\title{
Stability Analysis of the Heat Equation with Time-Delayed Feedback *
}

\author{
Sina Yamaç Çalışkan* Hitay Özbay ** \\ * Dept. of Electrical and Electronics Engineering Bilkent University, \\ TR-06800 Bilkent, Ankara, Turkey; e-mail: sina@bilkent.edu.tr \\ ** Dept. of Electrical and Electronics Engineering Bilkent University, \\ TR-06800 Bilkent, Ankara, Turkey; e-mail: hitay@bilkent.edu.tr
}

\begin{abstract}
In this paper we consider the heat equation with time delayed feedback. Recently, stability analysis of this system, with possibly time-varying delay, is done by Fridman and Orlov (2007, 2009); and a sufficient condition is obtained for stability in terms of a linear matrix inequality. Here we consider the same system, but with constant delay, and perform the stability analysis in the frequency domain. A necessary and sufficient condition is obtained in terms of the system parameters. The result is illustrated with numerical examples.
\end{abstract}

\section{INTRODUCTION}

In this paper we study the heat equation with time delayed feedback from input-output point of view. Inputs to the plant are boundary conditions at the end points of a one dimensional rod (the spatial variable $x$ takes values in $0 \leq x \leq \pi)$, and the output is the temperature at a point $x_{o} \in(0, \pi)$, denoted by $z\left(x_{o}, t\right)$, where $t$ represents the time. Transfer function of this system, without any feedback, is stable and has negative real poles $p_{n}=-n^{2}$, for $n=1,2,3, \ldots,[6]$. On the other hand, in the existence of a feedback, e.g. heat generation or loss, it is possible to destabilize the system, see e.g. [2]. In a series of papers, $[7,8,9]$, Fridman and Orlov investigated this phenomena for different types of time-delayed feedback configurations and they have obtained sufficient conditions for checking stability. In [9] time delay appearing in the feedback term is allowed to be time varying. In the present work, assuming that the time delay is constant, necessary and sufficient conditions are derived for checking stability in terms of the system parameters.

It should also be mentioned that systems represented by the heat equation, under different types of linear and nonlinear feedback, are studied by many researchers using various control techniques, see for example the recent papers $[2,4,5,11,12]$ and their references.

Rest of the paper is organized as follows. The system under consideration is defined in Section 2. By using a frequency domain analysis, location of the critical poles of the plant are studied and a stability condition is derived in Section 3 . Numerical examples are given in Section 4 and concluding remarks are made in Section 5.

\footnotetext{
* First author is supported in part by a scholarship from The Scientific \& Technological Research Council of Turkey (TÜBİTAK).
}

\section{PROBLEM DEFINITION}

The one dimensional heat equation is a partial differential equation which determines the temperature distribution on a rod:

$$
\frac{\partial}{\partial t} z(x, t)=a \frac{\partial^{2}}{\partial x^{2}} z(x, t)
$$

where $z(x, t)$ is the temperature of the rod (length $\pi$ ) at the point $x \in[0, \pi]$ at time instant $t \geq 0$. The parameter $a>0$ in the equation is a constant which depends on the conductivity of the medium. This system has been studied in the literature extensively with different types of initial and boundary conditions, see e.g. [6, 10].

The heat equation with feedback, $[1,4,5,9,11]$, takes the form

$$
\frac{\partial}{\partial t} z(x, t)=a \frac{\partial^{2}}{\partial x^{2}} z(x, t)+f(z)
$$

In this paper we consider a linear feedback in the form

$$
f(z)=a_{o} z(x, t)-a_{1} z(x, t-\tau)
$$

where $a_{0}, a_{1} \in \mathbb{R}$ are feedback gains and $\tau>0$ is the time delay, which is assumed to be a constant. In [9] the same problem is considered with time varying delay, and a sufficient condition for stability is derived in terms of a linear matrix inequality formed by the parameters $a, a_{0}, a_{1}, \tau$ and the upper bound of the derivative of $\tau$. In $[1,4]$ the feedback contains the non-delayed term only $f(z)=a_{0} z(x, t)$ and in [11] it takes the form $f(z)=$ $a_{0} z(x, t)+\frac{\partial}{\partial x} z(x, t)$. Other, possibly nonlinear, feedback forms are also considered in the literature, see e.g. [5].

We will assume zero initial conditions for the PDE, (2),

$$
z(x, \theta)=0 \quad \forall x \in(0, \pi) \text { and } \theta \in[-\tau, 0]
$$

and consider inputs at the boundary points 


$$
\begin{aligned}
& z(0, t)=u_{1}(t), \text { with } u_{1}(t)=0, \text { for } t<0 \\
& z(\pi, t)=u_{2}(t), \text { with } u_{2}(t)=0, \text { for } t<0 .
\end{aligned}
$$

Let the temperature at a point $x_{o} \in(0, \pi)$ is taken as the output of the system $y(t)=z\left(x_{o}, t\right)$. Transfer functions from $u_{1}$ and $u_{2}$ to $y$ are denoted by $G_{1}(s)$ and $G_{2}(s)$ respectively, and they are derived in the next section. In the next section, by analyzing the pole locations of $G_{1}$ and $G_{2}$ we obtain necessary and sufficient conditions for stability of this system in terms of the parameters $\left(a, a_{0}, a_{1}, \tau\right)$.

\section{STABILITY ANALYSIS IN THE FREQUENCY DOMAIN}

By taking the Laplace transform of

$$
\frac{\partial}{\partial t} z(x, t)=a \frac{\partial^{2}}{\partial x^{2}} z(x, t)+a_{o} z(x, t)-a_{1} z(x, t-\tau)
$$

with respect to the time variable we obtain

$$
\left(s-a_{0}+a_{1} e^{-\tau s}\right) Z(x, s)=a \frac{\partial^{2}}{\partial x^{2}} Z(x, s)
$$

where $s$ is the Laplace transform variable. Using the boundary conditions $Z(0, s)=U_{1}(s)$ and $Z(\pi, s)=U_{2}(s)$ we find the solution of $(5)$ as

$$
Z(x, s)=G_{1}(x, s) U_{1}(s)+G_{2}(x, s) U_{2}(s)
$$

where

$$
\begin{aligned}
& G_{1}(x, s)=\left(e^{-(x-\pi) \lambda(s)}-e^{(x-\pi) \lambda(s)}\right) / \Delta(s) \\
& G_{2}(x, s)=\left(e^{x \lambda(s)}-e^{-x \lambda(s)}\right) / \Delta(s)
\end{aligned}
$$

with

$$
\Delta(s)=e^{\pi \lambda(s)}-e^{-\pi \lambda(s)}
$$

and

Note that

$$
\lambda(s)=\sqrt{\frac{s-a_{0}+a_{1} e^{-\tau s}}{a}} .
$$

$$
G_{1}(0, s)=G_{2}(\pi, s)=1, \quad G_{1}(\pi, s)=G_{2}(0, s)=0,
$$

and $G_{1}\left(\frac{\pi}{2}, s\right)=G_{2}\left(\frac{\pi}{2}, s\right)$.

Locations of the zeros of the transfer functions $G_{1}\left(x_{o}, s\right)$ and $G_{2}\left(x_{o}, s\right)$ depend on the location of $x_{o} \in(0, \pi)$, where the output measurement is taken. Locations of the poles are the roots of the equation $\Delta(s)=0$, which is equivalent to

$$
e^{-2 \pi \lambda(s)}=1=e^{j 2 \pi n}, \quad n=0, \pm 1, \pm 2, \ldots
$$

or

$$
\lambda(s)= \pm j n \quad n=0, \pm 1, \pm 2, \ldots .
$$

Clearly $n=0$ is not admissible, because in this case (5) implies

and the solution is

$$
\frac{\partial^{2}}{\partial x^{2}} Z(x, s)=0
$$

$$
z(x, t)=u_{1}(t)+x\left(u_{2}(t)-u_{1}(t)\right) / \pi
$$

so (4) imposes conditions on free inputs $u_{1}$ and $u_{2}$; that makes the system ill posed. Thus, the poles of this system are the solutions $s \in \mathbb{C}$ satisfying

$$
\frac{s-a_{0}+a_{1} e^{-\tau s}}{a}=-n^{2}, \quad n=1,2,3, \ldots
$$

Putting $s=\sigma+j \omega$ and separating real and imaginary parts we obtain two equations for $\sigma$ and $\omega$ :

$$
\begin{aligned}
-a n^{2} & =\sigma-a_{0}+a_{1} e^{-\tau \sigma} \cos (\tau \omega) \quad n=1,2,3 \ldots \\
0 & =\omega-a_{1} e^{-\tau \sigma} \sin (\tau \omega) .
\end{aligned}
$$

\subsection{Stability Conditions}

By analyzing possible solutions of $(10)$ in $\mathbb{C}_{+}$we derive necessary and sufficient conditions for stability of (4). We begin with two remarks.

Remark 1. When $a_{1}=0$ the only solution of (12) is $\omega=0$ and thus the poles are at $\left(a_{0}-a n^{2}\right)$, for $n=1,2, \ldots$ In this case the system is stable if and only if $a>a_{0}$.

Considering the above observation, in the remaining part of the paper we will assume that $a_{1} \neq 0$ and $\tau>0$ (when $\tau=0$, clearly, $a_{1}$ and $a_{0}$ can be combined and the discussion of Remark 1 becomes valid).

Remark 2. Since a particular solution of (12) is $\omega=0$, all real poles $s=\sigma$ satisfy

$$
\sigma+a_{1} e^{\tau \sigma}=a_{0}-a n^{2}, \quad n=1,2,3, \ldots
$$

By the assumption that $a>0$, this equation has a solution for $\sigma \geq 0$ if and only if

$$
a-a_{0} \leq-a_{1}
$$

Therefore, the system is unstable if (13) holds.

In the light of Remark 2 we assume that $-a_{1}<a-a_{0}$, which is necessary for stability. Returning to (10) we see that the system is stable if and only if the roots of

$$
1+\frac{a_{1} e^{-\tau s}}{s+\left(a n^{2}-a_{0}\right)}=0
$$

are in $\mathbb{C}_{-}$for all $n=1,2,3, \ldots$, which is equivalent to stability of the feedback system shown in Figure 1, where

$$
G_{n}(s)=\frac{a_{1} e^{-\tau s}}{s+\left(a n^{2}-a_{0}\right)} .
$$

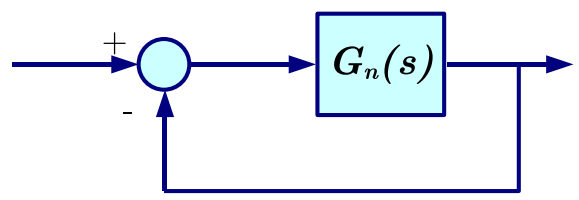

Fig. 1. Equivalent Feedback System.

A small gain argument can be used to show that the system is stable independent of delay if $\left(a n^{2}-a_{0}\right)>\left|a_{1}\right|$ for all $n=1,2,3 \ldots$ Clearly, if this condition is satisfied for 
$n=1$ then it is satisfied for all $n \geq 2$. Therefore, we conclude that the system is stable independent of delay if

$$
\left|a_{1}\right|<a-a_{0}
$$

Note that if $a_{1}<0$ and $-a_{1}<a-a_{0}$, then the system is stable independent of delay.

Thus, in order to make a stability analysis dependent on delay we assume that

$$
a_{1}>0 \text { and }-a_{1}<a-a_{0}<a_{1} .
$$

For a fixed $n$, we consider two cases for the stability analysis of the system shown in Figure 1:

Case 1: $0<a n^{2}-a_{0}<a_{1}$,

Case 2: $-a_{1}<a n^{2}-a_{0}<0$.

Before proceeding, we define

$$
\omega_{n}:=\sqrt{a_{1}^{2}-\left(a n^{2}-a_{0}\right)^{2}} .
$$

Note that $\omega_{n}$ is the frequency where we have

$$
\left|G_{n}\left(j \omega_{n}\right)\right|=1 \text {. }
$$

This observation will be used in phase margin computation for both cases.

Case 1. In this case $G_{n}$ is stable and stability of the feedback system shown in Figure 1 is equivalent to having a positive phase margin:

$$
\pi-\tan ^{-1}\left(\frac{\omega_{n}}{a n^{2}-a_{0}}\right)-\tau \omega_{n}>0 .
$$

The above condition can be expressed as

$$
\tau_{n}^{\max }:=\frac{\pi-\tan ^{-1} \sqrt{x_{n}^{2}-1}}{\left(a n^{2}-a_{0}\right) \sqrt{x_{n}^{2}-1}}>\tau
$$

where $x_{n}:=a_{1} /\left(a n^{2}-a_{0}\right)$.

Claim: $\tau_{n}^{\max }<\tau_{m}^{\max }$ when $n<m$.

Proof. See the journal version [3].

We conclude that the feedback system of Figure 1 is stable for all $n=1,2,3 \ldots$, if and only if it is stable for $n=1$, which is equivalent to

$$
\tau<\tau_{1}^{\max }=\frac{\pi-\tan ^{-1} \sqrt{x^{2}-1}}{\left(a-a_{0}\right) \sqrt{x^{2}-1}} \text { where } x=\frac{a_{1}}{a-a_{0}} .
$$

Multiplying both sides of the above inequality by $a_{1}$ we obtain the following simplified stability condition.

Lemma 1. The system (4) with $a_{1}>0$ and $0<a-a_{0}<a_{1}$ is stable if and only if

$$
a_{1} \tau<(\pi-\theta) / \sin (\theta) \text {, where } \theta:=\cos ^{-1}\left(\frac{a-a_{0}}{a_{1}}\right) \text {. }
$$

Case 2. Now consider $-a_{1}<a-a_{0}<a n^{2}-a_{0}<0$. In this case $G_{n}$ is unstable with one pole in $\mathbb{C}_{+}$. Stability of the feedback system shown in Figure 1 is equivalent to

$$
\tan ^{-1}\left(\frac{\omega_{n}}{a_{0}-a n^{2}}\right)-\tau \omega_{n}>0
$$

where the left hand side is the phase margin of the system. Similar to the analysis done in Case 1, define

$$
\tau_{n}^{\max }:=\frac{1}{a_{1}} \frac{\theta_{n}}{\sin \left(\theta_{n}\right)} \text { where } \theta_{n}:=\cos ^{-1}\left(\frac{a_{0}-a n^{2}}{a_{1}}\right) .
$$

So, stability in this case is equivalent to having $\tau_{n}^{\max }>\tau$ for all $n$ such that $-a_{1}<a n^{2}-a_{0}<0$. Again, it is a simple exercise to show that $\tau_{1}^{\max }<\tau_{n}^{\max }$ for all $n$ considered in this case. Therefore, we have the following result.

Lemma 2. The system (4) with $-a_{1}<a-a_{0}<0$ is stable if and only if

$$
a_{1} \tau<\theta / \sin (\theta), \quad \text { where } \theta:=\cos ^{-1}\left(\frac{a_{0}-a}{a_{1}}\right) .
$$

The results of both cases can be combined as follows. The system (4) with $a_{1}>0$ and $-a_{1}<a-a_{0}<a_{1}$ is stable if and only if $a_{1} \tau<\Omega\left(a-a_{0}\right)$ where

$\Omega\left(a-a_{0}\right):=\left\{\begin{array}{rl}\theta / \sin (\theta) & \text { if }-a_{1}<a-a_{0}<0 \\ (\pi-\theta) / \sin (\theta) & \text { if } 0<a-a_{0}<a_{1}\end{array}\right.$, and $\theta=\cos ^{-1}\left(\left|a-a_{0}\right| / a_{1}\right)$. When $a=a_{0}$ we have $\theta=\theta_{o}=\pi / 2$ and $\theta_{o} / \sin \left(\theta_{o}\right)=\left(\pi-\theta_{o}\right) / \sin \left(\theta_{o}\right)=\frac{\pi}{2}$. Therefore, the function $\Omega\left(a-a_{0}\right)$ is continuous around $a-a_{0}=0$.

In summary, the stability conditions derived can be restated as follows.

\section{Stability Conditions: The system (4) is}

(i) unstable independent of delay if $a-a_{0}<-a_{1}$,

(ii) stable if and only if $a_{1} \tau<\Omega\left(a-a_{0}\right)$ when $-a_{1}<a-a_{0}<a_{1}$,

(iii) stable independent of delay if $\left|a_{1}\right|<a-a_{0}$.

\subsection{Analysis of Pole Locations}

Note that $\Omega\left(-a_{1}\right)=1$ and $\Omega\left(a-a_{0}\right)>1$ for all parameters $a-a_{0}>-a_{1}$. Therefore, if $a_{1} \tau<1$, then the system is stable for all $a-a_{0}>-a_{1}$. When $a_{1} \tau \geq 1$ then we can perform an alternative stability analysis by searching for possible $\mathbb{C}_{+}$poles satisfying (11) and (12). For $\omega \neq 0,(12)$ is equivalent to

$$
e^{\tau \sigma}=\left(a_{1} \tau\right) \frac{\sin (\tau \omega)}{\tau \omega} .
$$

If $\left|a_{1} \tau\right| \geq 1$, then we may have a solution for (20) with $\sigma \geq 0$, that is

$$
\tau \sigma=\ln \left(\left(a_{1} \tau\right) \frac{\sin (\tau \omega)}{\tau \omega}\right)
$$

which is valid when

$$
\left(a_{1} \tau\right) \frac{\sin (\tau \omega)}{\tau \omega}>0 .
$$

Combining (11) and (12) we obtain

$$
\tau \sigma=\left(a_{0} \tau\right)-(a \tau) n^{2}-(\tau \omega) \cot (\tau \omega)
$$

Hence $\omega$ must satisfy

$$
\ln \left(\left(a_{1} \tau\right) \frac{\sin (\tau \omega)}{\tau \omega}\right)+(\tau \omega) \cot (\tau \omega)=\left(a_{0} \tau\right)-(a \tau) n^{2}
$$


for some $n=1,2,3 \ldots$ Note that if $\omega_{o}$ is a solution of (23), then so is $-\omega_{o}$, i.e. the poles appear in complex conjugate pairs, as expected. Above equations also show that the parameters $a, a_{0}, a_{1}$ and the variables $\sigma$ and $\omega$ can be scaled by $\tau$. So, we define

$$
\widehat{a}=a \tau, \quad \widehat{a}_{0}=a_{0} \tau, \quad \widehat{a}_{1}=a_{1} \tau, \quad \widehat{\sigma}=\tau \sigma, \quad \widehat{\omega}=\tau \omega
$$

and determine conditions under which $\widehat{\sigma} \geq 0$ can be a solution of the above equations.

Consider the system described by the transfer functions $G_{1}\left(x_{o}, s\right)$ and $G_{2}\left(x_{o}, s\right)$ with $x_{o} \in(0, \pi)$. Assume that $\widehat{a}_{1} \geq 1, a-a_{0}>-a_{1}$ and $a_{0} \neq a n^{2}$ for $n=1,2,3 \ldots$. Then, at least one pole of the system (4) is in $\overline{\mathbb{C}}_{+}$if and only if among all the solutions $\widehat{\omega}_{n, k}, k=0,1,2,3 \ldots$, of

$$
h(\widehat{\omega})=\widehat{a}_{0}-\widehat{a} n^{2} \quad n=1,2,3 \ldots
$$

where

$$
h(\widehat{\omega})= \begin{cases}\ln \left(\widehat{a}_{1} \frac{\sin (\widehat{\omega})}{\widehat{\omega}}\right)+(\widehat{\omega}) \cot (\widehat{\omega}) & \text { if } \widehat{a}_{1} \frac{\sin (\widehat{\omega})}{\widehat{\omega}}>0 \\ 0 & \text { if } \widehat{a}_{1} \frac{\sin (\widehat{\omega})}{\widehat{\omega}} \leq 0\end{cases}
$$

there exists at least one $\widehat{\omega}_{N, K}$ such that

$$
\widehat{a}_{1} \frac{\sin \left(\widehat{\omega}_{N, K}\right)}{\widehat{\omega}_{N, K}} \geq 1 \text {. }
$$

\section{NUMERICAL EXAMPLES}

Example 1. Consider the heat equation from [9]

$$
\frac{\partial}{\partial t} z(x, t)=\frac{\partial^{2}}{\partial x^{2}} z(x, t)+r z(x, t)+u(x, t)
$$

where $x \in(0, \pi)$ and $r$ is an arbitrary parameter satisfying $|r| \leq 1.9$. The feedback control is $u(x, t)=-z(x, t-\tau)$.

In terms of the parameters of (4) we have $a=1, a_{1}=1$ and $a_{0}=r$. From the stability conditions derived above we see that the stability region shrinks when $a-a_{0}$ gets smaller. Therefore, the worst case in this example is when $a_{0}=r=1.9$, i.e. when $a-a_{0}=-0.9$; since $\Omega(-0.9)=1.0347$ we conclude that this system is stable if and only if

$$
\tau<\tau_{\max }=1.0347 \mathrm{sec} .
$$

We can verify that for $\tau=1.0347$ the critical solution of (24) is $\widehat{\omega}=0.451$, and

$$
1 \times 1.0347 \times \frac{\sin (0.451)}{0.451}=0.9999785
$$

which says that stability is about to be violated for this value of $\tau$.

In [9], using the LMI toolbox of MATLAB, $\tau_{\max }$ is obtained as $\tau_{\max }=1.025 \mathrm{sec}$. Note that with $\tau=1.025$ the critical solution of $(24)$ is $\widehat{\omega}=0.4495$ and

$$
1 \times 1.025 \times \frac{\sin (0.4495)}{0.4495}=0.99083
$$

i.e., in this case, compared to $\tau=1.0347$, there is still some more room to increase $\tau$ before stability is violated.
The difference between $\tau_{\max }$ found here (which is exact) and the value obtained from [9] can be explained in two ways:

(a) the result of [9] gives a sufficient condition for stability, so there may be some conservatism in the LMI approach;

(b) it may be that the delay dependent LMI conditions of [9] are also necessary for this particular case, but the LMI toolbox tries to make a matrix negative definite (see equation (37) of [9]) and there is always some numerical freedom left in the parameter selection in this approach.

Example 2. As the second example, take $a=1, a_{0}=0$, $a_{1}=2$ and $\tau=1$. This corresponds to the problem studied in [8]. The graph of $h(\widehat{\omega})$ versus $\widehat{\omega}$ is as shown in Figure 2, where the intersection points with $\left(\widehat{a}_{0}-\widehat{a} n^{2}\right)$ are shown as the roots $\widehat{\omega}_{n, k}$ for $n=1,2,3$, and $k=0,1,2,3$.

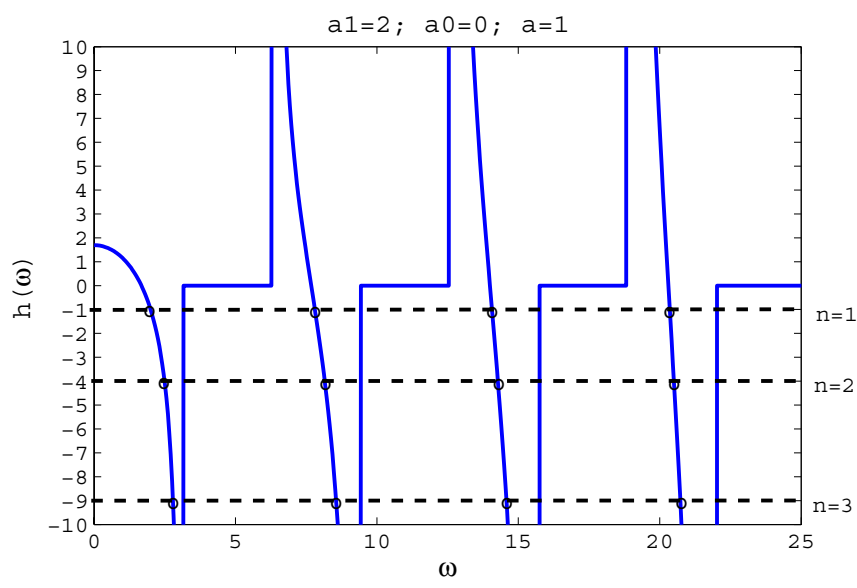

Fig. 2. The roots of $h(\widehat{\omega})=\widehat{a}_{0}-\widehat{a} n^{2}$.

First few roots are listed in the table below.

\begin{tabular}{|c|c|c|c|c|}
\hline \multicolumn{5}{|c|}{ The roots $\widehat{\omega}_{n, k}$} \\
\hline & $k=0$ & $k=1$ & $k=2$ & $k=3$ \\
\hline$n=1$ & 1.997 & 7.807 & 14.07 & 20.355 \\
\hline$n=2$ & 2.492 & 8.157 & 14.28 & 20.502 \\
\hline$n=3$ & 2.790 & 8.558 & 14.58 & 20.729 \\
\hline
\end{tabular}

\begin{tabular}{|c|c|c|c|c|}
\hline \multicolumn{5}{|c|}{$\sin \left(\widehat{\omega}_{n, k}\right) / \widehat{\omega}_{n, k}$} \\
\hline & $k=0$ & $k=1$ & $k=2$ & $k=3$ \\
\hline$n=1$ & 0.456 & 0.128 & 0.071 & 0.0490 \\
\hline$n=2$ & 0.243 & 0.117 & 0.069 & 0.0486 \\
\hline$n=3$ & 0.123 & 0.089 & 0.062 & 0.0460 \\
\hline
\end{tabular}

The maximum of the values $\sin \left(\widehat{\omega}_{n, k}\right) / \widehat{\omega}_{n, k}$ listed in the above table is 0.456 ; and we check that

$$
\widehat{a}_{1}=2<0.456^{-1}=2.193
$$

so the system is stable. Note that the right hand side of (25) does not correspond to the largest $\widehat{a}_{1}$ for which this system is stable. That can be found from the condition stated in Lemma 1 . In this case $0<a-a_{0}<a_{1}$ and 
$\theta=\cos ^{-1}(0.5)=\pi / 3$, so the system is stable if and only if

$$
a_{1} \tau=\widehat{a}_{1}<\frac{\pi-\theta}{\sin (\theta)}=\frac{\pi-(\pi / 3)}{\sqrt{3} / 2}=2.4184 .
$$

Indeed, if we let $a_{0}=0, a=1, a_{1}=2$ and $\tau=2.4184 / 2$ equation (24) has a solution $\widehat{\omega}=2.0944$, and

$$
2.4184 \frac{\sin (2.0944)}{2.0944}=1.0000
$$

which means that the system is unstable with a pole on the imaginary axis, at $\pm j \frac{\widehat{\omega}}{\tau}= \pm j 1.73205 \approx \pm j \sqrt{3}$.

\section{CONCLUSIONS}

An infinite dimensional system represented by the heat equation with a time delayed feedback term is studied. Necessary and sufficient conditions are derived for stability of this system by analyzing stability of an equivalent system in the frequency domain. For a given set of parameters $a, a_{0}, a_{1}$ and $\tau$ stability of the system can be determined from the conditions stated in Section 3.

Possible future studies include controller design for the plant whose transfer function, $\left[\begin{array}{ll}G_{1} & G_{2}\end{array}\right]$, is considered here. In order to design $H_{2}$ and $H_{\infty}$ controllers inner outer factorizations must be done; for this reason location of poles and zeros should be determined. Another problem left open is the approximation of this system by finite dimensional transfer functions. Finding approximations in the sense of $H_{\infty}$ will be useful in designing low order robust controllers for this plant.

\section{REFERENCES}

[1] D. M. Boskovic, M. Krstic, and W. Liu, "Boundary Control of an Unstable Heat Equation Via Measurement of Domain-Averaged Temperature," IEEE Trans. Automatic Control, vol.46 (2001), pp. 20222028.

[2] C. I. Byrnes, D.S. Gilliam, A. Isidori, V.I. Shubov, "Interior Point Control of a Heat Equation Using Zero Dynamics Design," Proceedings of the 2006 American Control Conference Minneapolis, Minnesota, USA, June 2006, pp. 1138-1143.

[3] S. Y. Çalışkan and H. Özbay, "Stability conditions for a system represented by the heat equation with time delayed feedback," submitted for publication, 2009.

[4] M-B. Cheng and W-C. Su, "A Proportional-plusintegral Boundary Control Design for an Unstable Heat Conduction System," Proceedings of the 46th IEEE Conference on Decision and Control, New Orleans, LA, USA, December 2007, pp. 4173-4178.

[5] J-M. Coron and E. Trelat, "Global steady-state controllability of one-dimensional semilinear heat equations" SIAM J. Control and Optimization, vol.43 (2004), pp. 549-569.
[6] R. F. Curtain and H. J. Zwart, An Introduction to Infinite-Dimensional Linear Systems Theory, Springer-Verlag, New York, 1995.

[7] Y. Orlov and E. Fridman, "On Exponential Stability of Linear Retarded Distributed Parameter Systems", Proceedings of the 7th IFAC Workshop on Time Delay Systems, Nantes, France, September 2007. (CDROM).

[8] E. Fridman and Y. Orlov, "On Stability of Linear Parabolic Distributed Parameter Systems with TimeVarying Delays", Proceedings of the 46th IEEE Conference on Decision and Control, New Orleans, LA USA, December 12-14, 2007, pp. 1597-1602.

[9] E. Fridman and Y. Orlov, "Exponential Stability of Linear Distributed Parameter Systems with TimeVarying Delays," Automatica, vol. 45 (2009), pp. 194201.

[10] J. J. Loiseau and H. Mounier, "Stabilisation de l'équation de la chaleur commandée en flux," ESAIM Proceedings, vol. 5 (1998), pp. 131-144.

[11] L. Qian and L. Tian, "Boundary Control of an Unstable Heat Equation," International Journal of Nonlinear Science, Vol.3 (2007), pp.68-73.

[12] S. Rao, V. Utkin, "Sliding Mode Control of OneDimensional Heat Exchange Processes," Proceedings of the 2006 International Workshop on Variable Structure Systems, Alghero, Italy, June 2006. 